\title{
Congenital myopia complicating pregnancy: A rare case and mini-review of the literature
}

\begin{abstract}
A highly myopic 35-year-old female patient (P0, G1) with reduced visual acuity R 3/10 $3 / 10$ due to bilateral choroidal neovascularization $(\mathrm{CNV})$ admitted at our Department. The patient had atomic history of congenital myopia firstly diagnosed at the age of 2-y-old.
\end{abstract}

The initial diagnosis established a refractive error over 8 diopters (high grade myopia) progressively increased.

During third trimester of gestation her vision was acutely diminished. The fundoscopic examination depicted elevated intraocular pressure and deterioration of myopia to $-30 \mathrm{D}$ bilaterally. (Fruoresceinangiography revealed active CNV).

Patient underwent selected caesarean section due to increased risk of macular hemorrhage. She discharged from hospital the $4^{\text {th }}$ pod in good clinical condition. Assiduous ocular and obstetrical monitoring appeared mandatory in order to insure optimal therapeutic mapping.

Objective of our study remains proper diagnosis and treatment of such a rare case.
Volume 12 Issue 3 - 202I

\author{
Papamargaritis Eftimios,' Papadaki Maria,' \\ Yperifanou Kyriaki, ${ }^{2}$ Sofoudis Chrisostomos' \\ 'Department of Obstetrics and Gynecology, Konstandopoulio \\ General Hospital Athens, Greece \\ 2Department of Ophthalmology, Konstandopoulio General \\ Hospital Athens, Greece
}

Correspondence: Dr. Chrisostomos Sofoudis, MD, PhD, MPH, Department of Obstetrics and Gynecology, Konstandopoulio General Hospital Athens, Ippokratous 209, I I472,Athens, Greece, Email chrisostomos.sofoudis@gmail.com

Received: May 02, 202I | Published: May 18, 2021

Keywords: high grade myopia, pregnancy, high intraocular pressure

\section{Introduction}

Myopia represents a very common refractive error among general population. The prevalence of myopia in Europe and USA estimated about $20-30 \%$ and its still increasing. ${ }^{1}$ Although in east Asia it reaches $80 \%$ among young adults, consisting the leading cause of blindness. ${ }^{2}$

This clinical entity consists a highly heritable entity with $80 \%$ of the cases reflecting genetic variety due to, in most of cases, refractive error. Approximately, one fifth of the myopic population presents with high myopia ( $\geq-6$ dioptres), a condition associated with vision impairment and complications such as retinal detachment, cataracts, glaucoma, myopic choroidal neovascularization $(\mathrm{CNV})$, foveoschisis, staphyloma, macular atrophy, and macular atrophy. ${ }^{2}$

Prevalence of myopia in normal term newborn estimates probably between $4 \%$ and $6 \%$, although less than $1 \%$ of this myopia diagnosed as high grade. Low grade myopia appears to be resolved or decreased over the first few months of life. Overall incidence appears to be increased with decreasing birth weight.

Many recent studies, with main objective refractive ocular vision, revealed premature infant with myopic or hyperopic ocular vision in contrast with normal term. ${ }^{3}$ In cases of congenital myopia proper diagnosis consists during the first neonatal three years.

It can be strongly associated with prematurity status or genetically formed, remaining in most of the cases clinical stable. Though, sometimes it appears as part of a syndrome such as deafness and myopia syndrome (DFNMYP), Stickler syndrome or Donnai-Barrow syndrome (DBS). ${ }^{4}$

Additionally, it requires appropriate surveillance and regular ocular evaluation in order to monitor potential complications from high myopia including cataracts, glaucoma and retinal detachment.

Choroidal neovascularization (CNV) represents a common vision-threatening ocular complication in patients with high myopia, affecting $5 \%$ to $11 \%$ of cases. Once confirmed as active CNV, timely treatment such as anti-VEGF therapy is recommended.
It involves the growth of new blood vessels which originate from choroid through a break in the Bruch membrane into the sub-retinal pigment epithelium (sub-RPE) or sub retinal space. During gestation, ocular morphology undergoes a number of hormone-induced hemodynamic changes. $\mathrm{CNV}$ develops in $5 \%$ of high myopic eyes and can be stimulated during pregnancy due to increased activity of angiogenic factors, including vascular endothelial growth factor (VEGF), erythropoietin (EPO) and placental growth factor (PIGF). ${ }^{5}$

Although the exact mechanism of action remains controversial, the activity of angiogenic factors can increase micro vascular permeability and fragility of new choroidal vessels, making prone to rupture during labor. During past years gynecologists and ophthalmologists shared the opinion that high grade myopia depicts a contraindication regarding spontaneous vaginal delivery due to its relation with rhegmatogenous retinal detachment.

Furthermore, presence of peripheral retinal degenerations, history of retinal detachment surgery, diabetic retinopathy, or glaucoma consist indications concerning termination of pregnancy. ${ }^{6}$ However, current bibliography offers no proof regarding correlation of spontaneous vaginal delivery with cases of retinal detachment.

This hypothesis was strongly supported by evidence that increased intraocular pressure during second stage of labor results in vitreous body which is being pressed against the retina, reducing the risk of retinal tears and retinal detachment. ${ }^{7}$

It is also not evidence-based that shortening the second stage of labor by performing instrumental delivery offers any benefit.

Despite those facts high grade myopia represents ultimate ocular indication for $\mathrm{CS}^{8}$ According to recent concluded studies, only indication for caesarian section (CS) consists myopic patients is the presence of $\mathrm{CNV}$, leading to sub retinal bleeding with acute visual loss.

It remains controversial whether glaucoma should be an indication for CS since Valsalva maneuver during normal vaginal delivery affects intraocular pressure (IOP). 
Valsalva maneuvers may cause IOP elevation and potential diminution of ocular perfusion. Nonetheless, similar concluded studies reveal no reduction of ocular perfusion during delivery. On the other hand, IOP increase during breath-holding sessions depicts no significant difference. Measuring of brief pressure fluctuations do not seem to be pathologic even for diagnosed damaged optic nerves. ${ }^{9}$

\section{Case report}

A highly myopic 35-year-old female patient (P0, G1) with reduced visual acuity R 3/1013/10 due to bilateral choroidal neovascularization (CNV) admitted at our Department. She had a history of congenital myopia firstly diagnosed at the age of two.
The initial diagnosis established a refractive error over 8 diopters (high myopia) progressively increased. Former gynecologic and atomic history was uneventful respectively.

During $20^{\text {th }}$ week of gestation her myopia was $-27 \mathrm{D}$ bilaterally with normal intraocular pressure and no active $\mathrm{CNV}$ or signs or retinal edema. Due to the absence of evidence for choroidal neovascularization $(\mathrm{CNV})$, a simple clinical and imaging surveillance was recommended.

Along third trimester of gestation her vision was acutely diminished. She was referred for further ocular evaluation. Fundoscopic examination depicted elevated intraocular pressure and deterioration of myopia to -30,97D right eye and -30,79D left eye. (Fruoresceinangiography revealed active CNV) (Figure 1) (Figure 2).

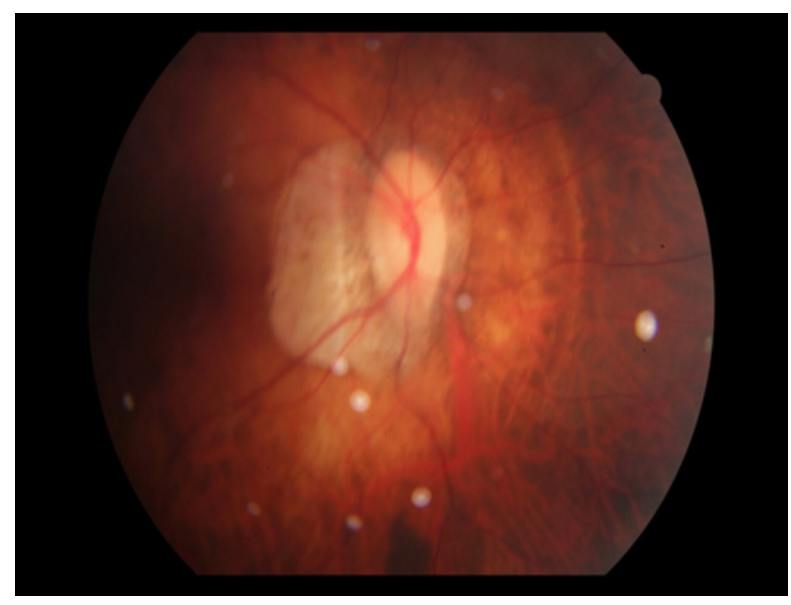

Figure I Fundoscopic evaluation. Myopic fundus, peripheral atrophy without sings of degeneration or vascularization of the macula, or other pathologic peripheral imaging findings.

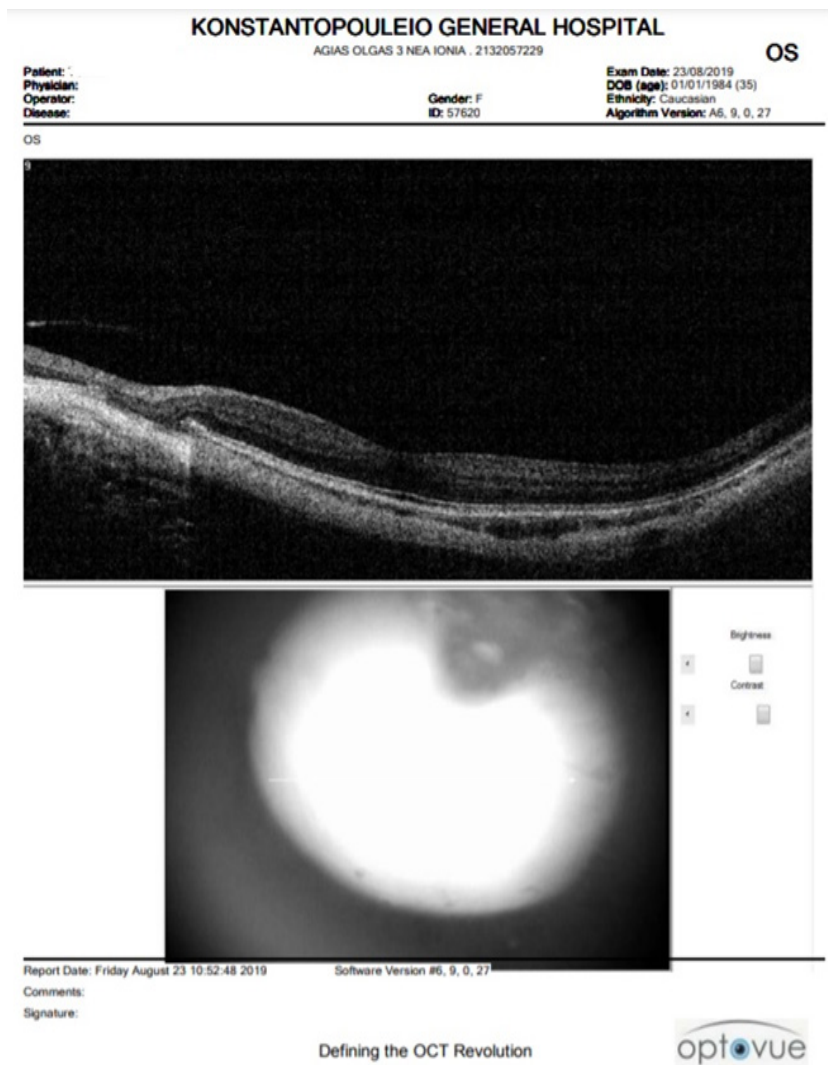

Figure 2 Optical Coherence Tomography (OCT) Peripheral atrophy, Macula normal, without rupture signs of pigment epithelium or Bruch membrane. Retinal attachment. 
Administration of anti- vascular endothelial growth factor (antiVEGF) agent as bevacizumab would be an optimal therapeutic strategy.

However, safety data on the use of anti-VEGF agents in pregnant patients are still limited and discussed controversially. ${ }^{10}$ Fetal growth monitoring along $28^{\text {th }}$ and $34^{\text {th }}$ week of gestation did not reveal any signs of pathology.

Patient underwent selected caesarean section (CS) at $39^{\text {th }}$ week of gestation due to increased risk of macular hemorrhage. She received spinal analgesia for the CS.

She delivered a healthy female new born with weight of $3200 \mathrm{~g}$ and Apgar Score 9 in the $1^{\text {st }}$ minute and 10 in the $5^{\text {th }}$ minute respectively.

Following current medical guidelines, whole surgical procedure was uneventful. Patient discharged from hospital the $4^{\text {th }}$ pod in good clinical condition.

\section{Discussion}

Pregnancy-associated ocular diseases have increased the level of difficulty concerning the pregnancy monitoring and mode of delivery among general population. Due to hematologic, hormonal, metabolic, cardiovascular, and immunologic procedures, pregnancy associated surveillance can be diagnosed as high risk.

Additionally, reflection of gestation pathophysiology on ocular vascular system still remains a controversial entity. Increased circulating blood volume and water retention can lead to corneal thickening, changing corneal curvature and consequently induce a temporary myopia and an associated distance vision impairment. ${ }^{11}$

Until early 90's high grade myopia (HM) consisted a predominant non-obstetrical indication for SC or instrumental delivery. Since then several conducted studies focused on pregnancy and delivery impact on ocular pathology remained controversial, depicting high grade myopia as indication for spontaneous vaginal delivery (SVD).

Subsequent studies revealed no significant adverse retinal changes such as rhegmatogenous retinal detachment or retinal degeneration after vaginal delivery, assuming preexisting ocular pathology as not a contra indication for instrumental or operative delivery.

High grade myopia complicating with $\mathrm{CNV}$ consists a subject of current research. Myopic patients with concomitant choroidal neovascularization in the macular region reflect a high risk of retinal hemorrhage during second stage of labor and subsequently a possibility of a non-reversible sudden deterioration of visual acuity.

Minor conducted studies cannot establish a safe mode of delivery, whether performing NVG in a patient with CNV. Although there is a current conflict on that matter, $\mathrm{CNV}$ consists an individual nonobstetric indication for CS focusing on ultimate quality of maternal and fetal life.

\section{Conclusion}

Throughout current bibliography, any randomized trials can establish pathophysiology of pregnancy and co-existing ocular disease.
Few of them, originated most of them from Eastern Europe referred to a small number of patients inadequate to assess potential effects of pregnancy and labor on visual function, providing firm conclusions.

In rare and unpredictable conditions, such our case, assiduous patient counseling and pregnancy surveillance can lead to ultimate therapeutic mapping.

\section{Acknowledgments}

None.

\section{Funding}

None.

\section{Conflicts of interest}

All authors declare any financial interest with respect to this manuscript.

\section{References}

1. Morgan IG, French AN, Ashby RS, et al. The epidemics of myopia: Aetiology and prevention. Prog Retin Eye Res. 2017;62:134-149.

2. Wu PC, Huang HM, Yu HJ, et al. Epidemiology of myopia. Asia Pac J Ophthalmol (Phila). 2016;5(6):386-393.

3. Holmström G, Azazi M el, Kugelberg U, et al. Ophthalmological long term follow up of preterm infants: a population based, prospective study of refraction and its development. Br J Ophthalmol. 1998;82(11):1265-1271.

4. Ordonez JL, Tekin M. Deafness and myopia syndrome. University of Washington, Seattle, Seattle (WA); 2017.

5. Ghaem-Maghami S, Cook H, Bird A, et al. High myopia and preeclampsia: a blinding combination. BJOG. 2006;113(5):608.

6. Mohammadi SF, Mojgan LN, Ashrafi NE, et al. A survey of ophthalmologists and gynecologists regarding termination of pregnancy and choice of delivery mode in the presence of eye diseases. $J$ Curr Ophthalmol. 2017;29(2):126-132.

7. Kuba GB, Kroll P. Labor monitoring and indications for abortion and cesarean section in eye diseases - an overview. Klin Monatsbl Augenheilkd. 1997;211:349-353.

8. Coşkun B, Serdar O, Buğra C, et al. Retrospective evaluation of indications and birth results of cesarean section due to ophthalmologic diseases. The European Research Journal. 2020;6(4):287-291.

9. Lam AK, Lam CH. Effect of breath-holding on pulsatile ocular blood flow measurement in normal subjects. Optom Vis Sci. 2004;81:597-600.

10. Farzan K, Heshmatollah G, Zahra N, et al. Intravitreal vascular endothelial growth factor (VEGF) inhibitor injection in unrecognized early pregnancy. Invest New Drugs. 2016;34(5):650-653.

11. Grabowska M, Ciszewska J, Godowska J, et al. Delivery in myopic women: a comparison of mode of delivery in years 1990, 2000, and 2010. 2019;25:7715-7719. 